\title{
A logical type discriminant model for profiling a segment structure
}

Received (in revised form): 25th February, 2003

\section{Margarida G. M. S. Cardoso}

is an assistant professor at ISCTE-Instituto Superior de Ciências do Trabalho e da Empresa, Lisbon (Portugal). She holds a degree in mathematics from the University of Lisbon. She is a Master of Operations Research and Systems Engineering and holds a PhD in systems engineering, both from the Technical University of Lisbon. Her research domains include multivariate statistical and machine learning methods in marketing research.

\section{Luíz Moutinho}

is a foundation chair of marketing in the Department of Business and Management, University of Glasgow. He is currently the Director of the Doctoral Programmme at the University Business School.

\begin{abstract}
Profiling a segment structure may help to make segments accessible and generally contribute to improving the understanding of the segments, thus helping to support management decisions. Logical type discriminant models yield results that are easy to understand; this makes them particularly useful for profiling. From this point of view, the induction of propositional rules yields better results when compared with hierarchical structures derived from decision tree algorithms. An application in the tourism market illustrates some advantages of the C5 algorithms for the induction of classification trees and sets of propositional rules for profiling a segment structure.
\end{abstract}

Margarida Cardoso

Department of Quantitative Methods, Instituto Superior de Ciências do Trabalho e da Empresa, Av. das Forças Armadas, 1649-083 Lisboa, Portugal.

Tel: + 351 217903941;

e-mail:

margarida.cardoso@iscte.pt

\section{INTRODUCTION}

Profiling a segment structure (typically a consumers partition) is meant to provide better understanding of segments, finding the characteristics that discriminate them. Additionally, it may also yield means to classify individuals into the segments.

In 1978, Wind had already identified the problem of determining segment membership (classification) and profiles (discrimination) as a 'major

consideration involved in segmentation research studies'. ${ }^{1}$ Wedel and Kamakura point out the importance of profiling 'with consumers (firms) descriptors to fulfil the accessibility requirement for effective market segmentation'.2 Several authors $^{3-5}$ recognise the importance of profiling in the particular context of tourism applications. Mazanec ${ }^{6}$ underlines the need to search for additional characteristics generating more complete segment profiles, emphasising that a market segmentation strategy is likely to fail unless segments are described objectively, namely with additional geographical, demographic, socio-economic, behavioural and lifestyle criteria. Andereck and Caldwell $^{7}$ emphasise that the segments' characteristics may help marketers to tailor and promote a product or service more effectively. Bargeman et al. ${ }^{8}$ point out that managers are interested in the classification of tourists according to socio-economic variables as it allows them to target potential customers better. 
Table 1: Some profiling applications

\begin{tabular}{ll}
\hline Alternative profiling methods & Examples of applications \\
\hline Linear discriminant analysis & Rao and Wang, $1995^{9}$ \\
Logistic regression & Bucklin and Gupta, $1992^{10}$ \\
Covariates model & Dillon, Kumar and Borrero, $1993^{11}$ \\
& Gupta and Chintagunta, 1994 ${ }^{12}$ \\
Neural networks & Mazanec, $1992^{13}$ \\
Recursive based partitioning (CHAID) & Baron and Worral, $1996^{14}$ \\
\hline
\end{tabular}

Several methodologies can be considered for profiling. Table 1 illustrates some of these with examples of applications. In general, profiling a segment structure can be viewed as a discriminant and classification data analysis task. It is a dependence analysis that considers a segment structure as the dependent variable and several relevant variables for profiling as independent. These descriptors should be combined in some way (functional, logical, graphical or other) in order to discriminate better among the segments, yielding a better understanding of the differences between them. Classification can be seen as the conclusion of discriminant analysis, as discriminant rules may be applied to allocate individuals into the segments.

The most commonly used statistical approaches for discriminant analysis are linear discriminant analysis and logistic regression. Linear discriminant analysis is the most frequently used method for profiling. ${ }^{15}$ However, the assumptions of this analysis are seldom met as it assumes (for example) that descriptors (discriminant variables) have normal distributions. Logistic regression is an alternative approach for classification when normality assumptions are not met. $^{16}$

Bucklin and Gupta developed a nested logit latent segments model to derive households segments that were subsequently characterised by a logistic regression model. ${ }^{17}$ Households were assigned to segments by using their posterior probabilities of segment membership based on their purchase histories. Segments showed different responses concerning choices and purchases of liquid laundry detergents. Profiling of a four segment structure (two choice segments with two nested incidence segments each) was based on demographic and purchase behaviour variables. Four binary logistic regressions were conducted to this end, the emphasis being placed on characterisation and not on classification.

An alternative approach that may be considered for profiling is the inclusion of covariates (or concomitant variables) in a latent segments model. A reparameterisation of posterior probabilities of latent segments is a means to include profiling variables (covariates) in the estimation of the model. ${ }^{18}$ Naturally the application of this method implies the use of a mixture model for performing segmentation.

The increase in the availability of large amounts of information supports the use of machine learning techniques to perform discriminant analysis. Using the machine learning terminology, this is a supervised learning task, as segments (dependent variables) are already known (as a result of previous segmentation) and this knowledge supervises learning of discriminant and classification rules based on explanatory variables.

Mazanec compared the performances of a neural network algorithm (a 
machine learning methodology) and linear discriminant analysis to predict segment membership. Both approaches provided classification of tourists into benefit segments, derived by a non-hierarchical clustering algorithm. The descriptor variables used concern demographic, socio-economic and behavioural attitudes. Similar hit rates (proportions of individuals correctly allocated to the segments) resulted from the two approaches. The author pointed out, however, some advantages of the neural network approach considering it as "neither restricted by the number of predictors nor by the number of segments' and 'much more flexible than conventional multivariate techniques with respect to incomplete or missing data'. ${ }^{19}$ In fact, these may be considered advantages of machine learning methodologies for classification when compared to traditional multivariate statistical approaches (eg linear discriminant analysis or logistic regression analysis) relying on complete observations and for estimation purposes and having difficulty dealing with large numbers of variables. $^{20,21}$

Besides the neural network approach other machine learning methods may be considered for profiling, in particular those providing logical type discriminant models. In addition to being able to deal with many and different types of variables and to overcome the difficulties due to the presence of missing values, these algorithms generate interpretable discriminant rules, which is considered relevant for the task of profiling. Several algorithms providing logical type discriminant models, models yielding logical type rules for segments characterisation (eg 'if age $>35$ then individual belongs to segment $\mathrm{X}$ ') may be considered for profiling. Among them the Chi-Square Automatic Interaction Detection (CHAID) ${ }^{22}$ and Classification and Regression Trees (CART) ${ }^{23}$ are the best known. Baron and Worrall used the CHAID algorithm to profile two binary segment structures. They were particularly interested in profiling a segment of individuals who tended to purchase less healthy foods. They also profiled customers that tended to develop customer-to-customer interactions during purchase (included in the second segment structure). Profiling was based on demographics for the former case and also included behavioural variables in the latter. CHAID provided clear and easy to understand tree diagrams, each terminal node of the trees (subset of individuals) being associated with a propositional condition (specific descriptor values). ${ }^{24}$

Bargeman et al. used CHAID as a means to overcome the problem associated with the existence of many missing values when trying to consider several variables for profiling. Specifically, the authors used CHAID to find a categorisation of profiling (socioeconomic) variables and subsequently a loglinear logit analysis was performed to estimate the effects of the socio-economic variables on the probability of belonging to a segment..$^{25}$ Another author suggested the use of a decision tree to help support the interpretation of logistic regression results. In this approach a CHAID regression tree was adopted to explain probabilities estimated by the logistic model, considering the same descriptors as logistic regression. ${ }^{26}$

The main goal of this paper is to illustrate the utility of classification trees and sets of propositional rules as alternative methods for profiling a segment structure. The use of the C5 classification tree algorithm ${ }^{27}$ is suggested, as it provides discriminant logical type models implementing new techniques which may be advantageous when compared with similar approaches (eg 
CHAID or CART). Furthermore, the use of C5 specific algorithm for the induction of propositional rules is advocated as it yields results that are very easy to understand.

An application referring to the segmentation of clients of a hotel chain illustrates the proposed approach.

\section{METHODOLOGICAL APPROACH}

\section{Measures and tests of association}

Measuring the degree of association between candidate profiling variables and the segments may be considered a preliminary step in the task of profiling. For example, the degree of association between the segments and a nominal variable may be measured by the $\mathrm{V}$ Cramer Statistic (based on the chi-square statistics). Another measure of association, the uncertainty coefficient $U$ that is based on the measure of entropy, provides additional information about the importance of a certain nominal attribute to explain the segments. Both the $\mathrm{V}$ Cramer statistic and the uncertainty coefficient, $\mathrm{U}$, assume a zero value if there is no association and the value one when the degree of association is maximal. ${ }^{28}$

The use of statistical inference is needed when trying to establish that the association between one specific descriptor and the segments is significant. The Pearson chi-square test, for example, may be used for this end, being able to infer the association between one nominal variable and the segments. For a quantitative variable, the analysis of variance (ANOVA) test may be used to evaluate the significance of the difference of the variable means for the various segments, although certain assumptions must be met (eg normality). If assumptions are not met, nonparametric tests $^{29}$ like the Kruskal-Wallis test may be needed to infer the association between ordinal or metric profiling variables and the segments.

As a result of this preliminary analysis, variables yielding a significant association with the segments may be used for the construction of a model that discriminates between the segments. In particular, a logical type discriminant model may be considered to explain the differences between the segments and help to classify new individuals into the segments.

\section{Logical type discriminant models}

The C5 algorithm ${ }^{30}$ provides the induction of logical type discriminant models, namely classification trees and sets of propositional rules.

\section{Classification trees}

Classification trees translate a hierarchical partitioning process that starts with a sample of observations concerning descriptors and segment membership (root node) and successively divides nodes based on the descriptors' values. This process attempts to decrease diversity in each new descendent node of the tree, in order to decrease the risk of classification. Thus, in a classification tree each terminal node (leaf) represents a set of relatively homogeneous observations in what concerns segment membership, the class distribution tending to concentrate in one particular segment.

The C5 algorithm grows a classification tree based on the information gain ratio measure, an entropy-based criterion. According to this criterion, the selection of a specific predictor $X_{j}$ to split a node $\mathrm{O}$ is guided by the objective of decreasing diversity (entropy) in the descendent nodes. Diversity in these child nodes should then be inferior to diversity in the parent 
node $\mathrm{O}$. As a consequence, the frequency distribution in each child node will thus tend to concentrate in specific segments.

In other words, splitting a node $\mathrm{O}$ based on a chosen attribute $X j$ (and on a particular categorisation of $X j$ 's levels) translates the capacity of the chosen attribute to add information concerning classification of individuals of node $\mathrm{O}$ into the segments.

If $X_{j}$ is metric or ordinal, binary partitions will be considered as candidates (thresholds will be the sorted values of $X_{j}$ ). If $X_{j}$ is categorical, its categories will yield a partition of node $O$. As an option, $\mathrm{C} 5$ provides a heuristic procedure that considers possible combinations of attributes' categories in order to optimise the information gain ratio.

To deal with missing observations concerning a chosen attribute $\left(X_{j}\right)$ to split node $\mathrm{O}, \mathrm{C} 5$ divides these observations among the descendent nodes of $\mathrm{O}$, according to the empirical distribution of frequencies associated with the correspondent categorisation (partition) $a^{*}$.

C5 provides pruning of the initial classification tree to avoid overfitting. A descendent subtree $A_{0}$ of node $O$ will be pruned if its pessimistic error of classification is greater than the one corresponding to node $\mathrm{O}$ (pessimistic error being the upper bound of a confidence interval associated with the proportion of incorrectly classified observations).

The final classification tree may be translated in a set of propositional rules, each one corresponding to a leaf node. In general, a propositional rule is represented by an 'if condition then class' logical type expression where the condition is a conjunctive proposition that allocates specific values to the descriptors. In particular, in a classification tree, each rule's condition (descriptors' values) is obtained following the path from the root to each terminal node and the rule's class is the modal segment for this leaf.

\section{Propositional rules}

A set of propositional rules may provide means to classify observations that meet the conditions associated with each class. It may be derived directly from a classification tree or a specific algorithm for the induction of propositional rules may be used, yielding discriminant models that cannot be translated into trees. After deriving the classification tree, C5 provides means to simplify, reduce and rank the initial set of propositional rules that corresponds to the leaves of the classification tree, yielding a new model of classification. Simplification of the initial subset of rules obeys the minimum pessimistic error criterion, trying to generalise rules by removing propositions from the rules' conditions (pessimistic error is considered to compare precision of alternative rules).

After simplification, a simulated annealing heuristic is applied to each subset, discarding some rules in order to minimise an estimate of the number of bits necessary to encode each subset (minimum description length criterion).

Finally, rules within each subset are sorted by precision and subsets are sorted according to a minimum of false positives criterion. As such, subsets that incorrectly classify the least number of cases are ranked in first place.

Classification of an observation $x_{\mathrm{i}}$ within a class (segment) is finally provided by the first rule that covers it. If it is covered by multiple rules corresponding to different classes, voting takes place, the weight associated with each vote being the precision associated to each rule. When $x_{\mathrm{i}}$ is not covered by any rule, a default classification is used and $x_{\mathrm{i}}$ is allocated to the segment that 
contains the most training cases not covered by any rule.

C5 may also incorporate costs of incorrect classification of an observation that belongs to a class $1^{*}$ in a class 1 of the dependent variable $\left(1,1^{*} \in\{1 \ldots S\}\right)$, in the final classification decision. In this case a minimum cost criterion is adopted (instead of maximum precision).

Finally, in addition to the original software implemented by Quinlan, programs that may be used to run C5 (classification trees and propositional rules) are included in some well-known data mining and statistical packages (eg the SPSS Clementine version was used in the present work).

\section{Model fitting}

\section{Measures of precision}

Classification trees and propositional rules may be associated with the corresponding measures of precision for classification. In particular, hit rates (the proportion of correctly classified observations) returned by estimated models may illustrate model fitting. Furthermore, these measures may be compared with default measures of classification precision (eg precision that derives from allocating all individuals to the larger segment, according to a majority rule).

\section{Using test samples}

When using hit rates corresponding to the training sample there is a tendency to overestimate precision, as the training model tends to overfit this sample's specific set of observations. To overcome this problem, the model derived from the training sample may be applied to a test (or holdout) sample in order to obtain more realistic estimates of classification precision. Furthermore, this procedure will help to test the model's consistency, which may fail to be proved if estimates derived from the training and test samples are very different.

Increasing the sample size may be a way to improve the precision and consistency of one model because, in general, larger samples tend to be more representative of the population. For this reason, training samples of several hundreds or thousands of cases are commonly found in practical applications, typically accounting for around 60 per cent to 70 per cent of the original sample. In fact, if a good pattern is not discovered (with an associated good level of precision when it refers to classification) or the good pattern may not be replicated (eg using a test sample) it may be that the original sample was not large enough. However, it is also necessary to be aware that even very large samples may not be representative or that, even so, patterns may be difficult to find (eg they may not exist).

\section{Interpretability}

Finally, the interpretability of the segment structure profiles yielded by each logical type discriminant model (classification tree or set of propositional rules) is discussed, helping to support model adequacy.

\section{APPLICATION}

\section{Introduction}

In the present application the segments of clients of a hotel chain are considered for profiling. Segmentation is based on behavioural variables that provide more meaningful segments from the management perspective. Demographic variables are used to profile segments, aiming to identify and access them, the resulting logical type characterisation being expected to support targeting. 
Table 2: Segment structure and segmentation base

\begin{tabular}{llll}
\hline & First time users & Regular users & Heavy users \\
Total & 442 & 1347 & 424 \\
percentage & 20.0 & 60.9 & 19.2 \\
\hline & Base variables & & \\
& Mean & & 4.2 \\
\hline B & 0.4 & 1.4 & 6.4 \\
C & 0.2 & 1.9 & 2.0 \\
CSUP & 0.1 & 0.8 & 5.5 \\
CH & 0.2 & 1.8 & 18 \\
Total (to the nearest & 1 & 6 & \\
whole figure) & 1 & &
\end{tabular}

\section{Database}

The data arose from the answers to a questionnaire directed to the Portuguese clients of Pousadas de Portugal, a high-end small hotel chain. The questionnaires were distributed in all hotels (42 at the time) between November 1996 and October 1997. The distribution was organised following a quarterly plan, each quarter including an equivalent group of heterogeneous pousadas. At the time of data collection there were four types of pousadas - B, $\mathrm{C}$, CSUP and $\mathrm{CH}$ - roughly corresponding to increasing average prices. (Pousadas $\mathrm{CH}, \mathrm{CSUP}, \mathrm{C}$ and $\mathrm{B}$ were in number, 13, 4, 14 and 11, respectively).

The sample collected under this project includes over 2,500 respondents (global reply rate was 74 per cent). Information about the respondent's profile and the evaluation of the stay is included in the database.

\section{Segment structure}

The segments of Portuguese clients of the pousadas are derived through a mixed process. First time users of pousadas constitute an a priori segment, as the specific characteristics of this segment were a priori found interesting to explore. The segmentation of the remaining clients of pousadas is performed through a $\mathrm{K}$-means procedure.
Base variables for segmentation $(\gamma)$ express stay frequency and revealed preferences, measured by the number and type of pousadas where the respondents had already stayed overnight. $\left.Y_{1}=\mathrm{B}\right)$ refers to the number of $\mathrm{B}$ type pousadas; $Y_{2}=\mathrm{C}$ ) refers to the number of $\mathrm{C}$ type pousadas; $Y_{3}=\mathrm{CSUP}$ ) refers to the number of CSUP type pousadas; $\left.Y_{4}=\mathrm{CH}\right)$ refers to the number of $\mathrm{CH}$ type pousadas. As frequency of use is the general base for segmentation, segments are named accordingly as regular users and heavy users of pousadas (see Table 2). Regular users stayed in around six pousadas and heavy users in about 18 (average totals).

\section{Profiling}

Demographic variables are generally more reliable characteristics than alternative segment descriptors such as attitudes or psychographics. ${ }^{31}$ Consequentially, these variables in particular, and observable variables in general, are often used for profiling a segment structure, helping to identify and to provide access to the segments. ${ }^{32}$ When considering a particular application, the selection of variables for profiling is, however, problem and data dependent.

In the present application, the demographics available for profiling the segments of the Portuguese clients of 
Table 3: Demographics variables used in profiling

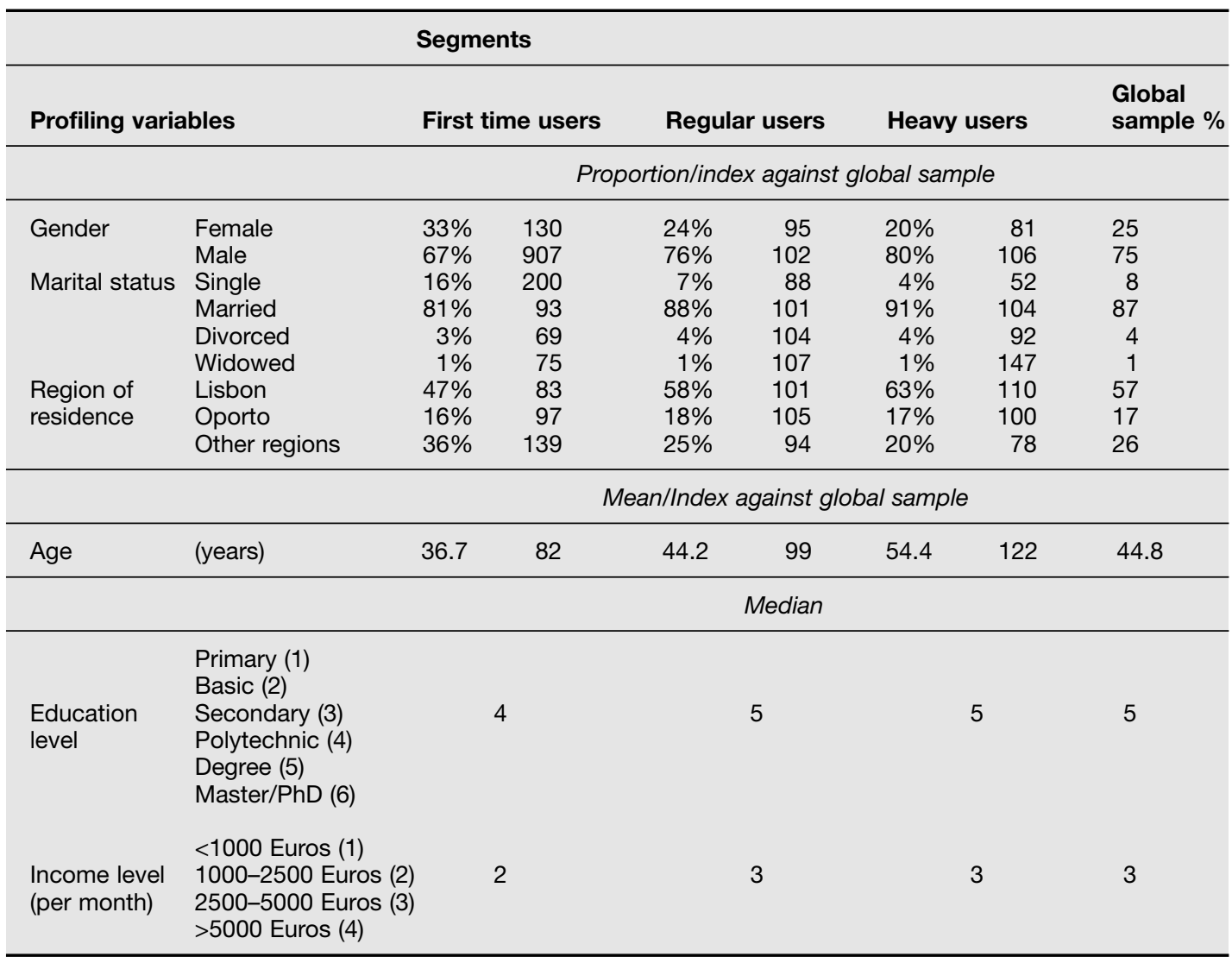

pousadas are presented in Table 3 - the table also includes some descriptive measures for each segment.

According to the results of association tests (see Table 4), there are significant differences between the segments and all the demographic descriptors

(Kruskal-Wallis tests provide conclusions for the metric descriptors and chi-square tests provide evidence of significant associations between the segment structure and categorical descriptors). Thus, all are considered for the profiling analysis.

In order to profile first time, regular and heavy users the induction of classification trees, as well as sets of propositional rules (based on the C5 algorithms) are used (see Figure 1).

Two random subsamples from the original sample are generated: one for estimating the discriminant models (the training sample, corresponding to 65 per cent of the global sample) and the other for obtaining hit rates estimates (the test sample). The choice of a larger training than test sample favours the learning phase, hoping to obtain a model that performs better in a test (holdout) sample.

The C5 algorithm is first run to provide a classification tree (C5 CT). The corresponding parameters are indicated in Table 5. The tree is first allowed to grow almost complete (a minimum number of two records per branch is considered) and then pruning is used in order to favour interpretation of results and to prevent overfitting.

According to the results provided by 
Table 4: Association test results

\begin{tabular}{llllll}
\hline Kruskal-Wallis tests & \multicolumn{2}{l}{ d.f } & Chi-square tests & d.f \\
\hline Age & 336.44 & 2 & Gender & 17.21 & 2 \\
Education level & 47.82 & 2 & Marital status & 44.78 & 6 \\
Income level & 125.84 & 2 & Region & 29.37 & 4 \\
\hline
\end{tabular}

Note: All tests return significant associations with the segments $(p<0.0001)$

Table 5: Algorithms' parameterisations

\begin{tabular}{|c|c|c|c|}
\hline & \multicolumn{3}{|c|}{ Recursive based partitioning algorithms } \\
\hline & $\begin{array}{l}\text { Classification } \\
\text { tree (C5 CT) }\end{array}$ & Rules (C5 Ra) & Rules (C5 Rb) \\
\hline Pruning & & $75 \%$ & \\
\hline Minimum records per branch & & 2 & \\
\hline Group symbolic values & & Yes & \\
\hline Costs of incorrect classification & unit & unit & $\mathrm{C}($ Regular $\mid$ Heavy $)=2$ \\
\hline
\end{tabular}

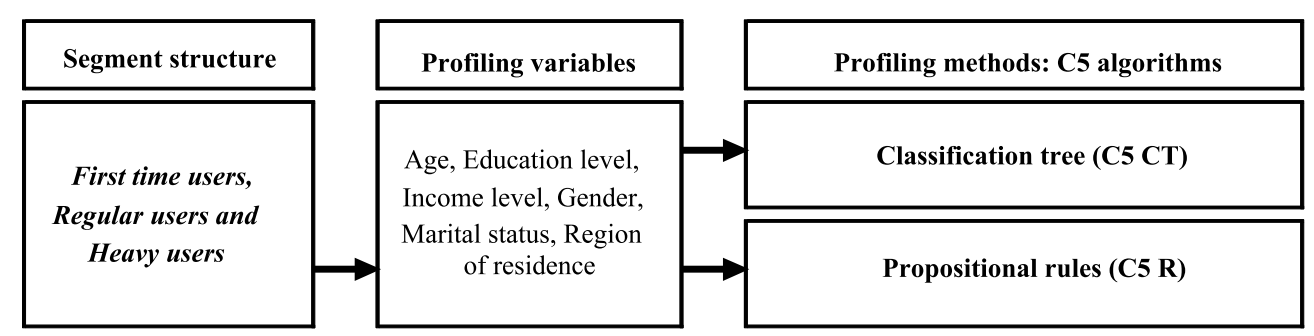

Figure 1 Profiling methods

this model (C5 CT), 62 per cent (estimate corresponding to the test sample) of the clients are correctly classified. The numbers in the leaves of the classification tree (see Figure 2) illustrate the precision of the corresponding rules, eg 'if Age $>27$ and Education level $\leq 4$ and Income level $\leq 1$ then first time users' is a rule that covers around 15 cases (note that C5 fractionates missing cases) and this rule classifies correctly 77.3 per cent of the cases it covers (cases that match its condition).

The induction of a set of propositional rules is also used to provide means to discriminate among first time, regular and heavy users of pousadas. Two alternative propositional rules models are considered for this purpose (C5 Ra and C5 Rb), its parameters being presented in Table 5 .

The first model's results are illustrated in Table 6, its precision being the same as the one obtained for the corresponding classification tree (a 62 per cent hit rate referred to the test sample).

As C5 CT and (consequently) C5 Ra do not provide rules for the heavy users segment, an alternative model (C5 Rb), which associates a higher cost of incorrect classification if heavy users are classified as regular users (see Table 5) is proposed. Although this model favours correct classification of heavy users, its results are more difficult to interpret (see Table 7). 


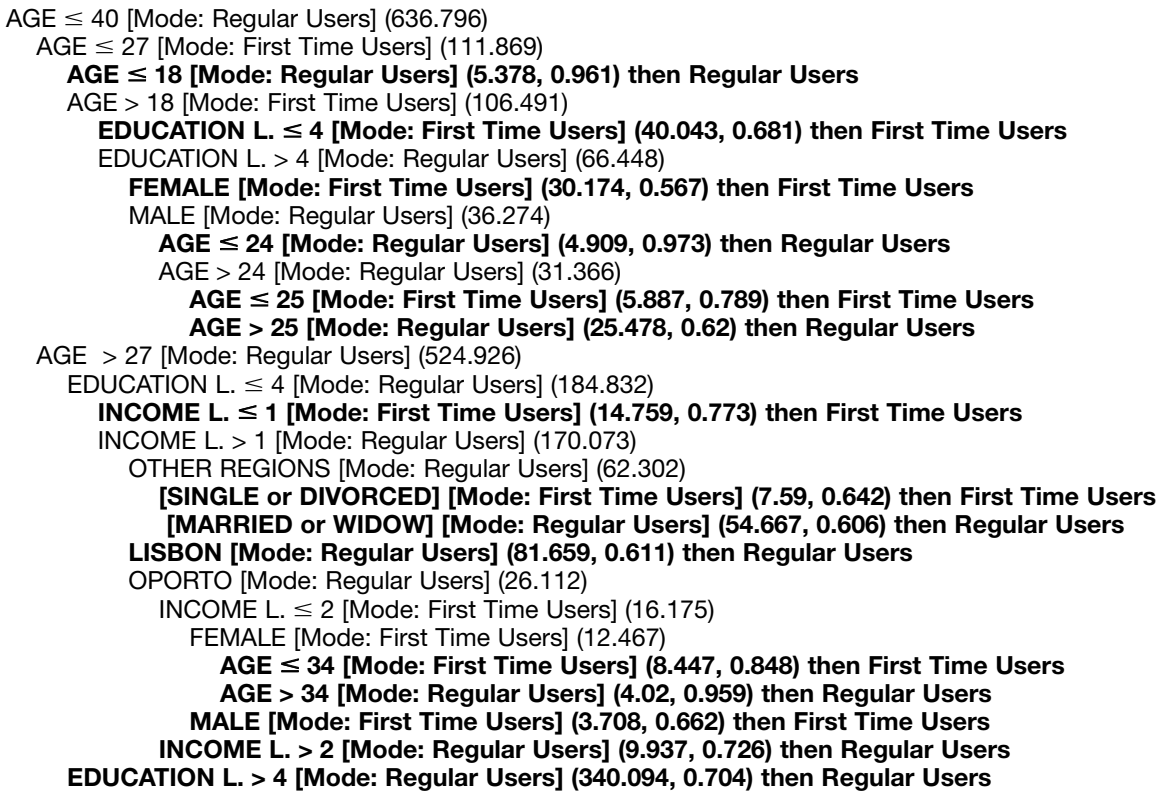

Note: the terminal nodes (leaves) of the tree are signed in bold and the number and proportion of correctly classified cases are presented between parenthesis.

Figure 2 Classification tree (C5 CT)

In conclusion, model C5 Ra provides some rules having a fairly high precision and adds insights concerning the segments; eg the rules 'if Age $\leq 40$ and Education level $\leq 4$ and Income level $\leq 1$ then first time users' and 'if Other Regions and Age $>67$ and Educational level $>2$ then heavy users' may suggest that the individuals that had not yet been in a pousada tend to be younger and with lower academic and income levels, and that the individuals living outside Lisbon and Oporto tend to be heavy users if they are older and have higher educational levels.

Regarding the obtained results' interpretability it may be concluded that the sets of propositional rules are generally easier to interpret, when compared with the original classification trees (see Figure 2 and Table 6), because:
- C5 $\mathrm{R}$ rules have no hierarchical structure in opposition to C5 CT rules

- the conditions in the $\mathrm{C} 5 \mathrm{R}$ rules have fewer propositions which makes them simpler

- the number of rules derived from C5 $\mathrm{R}$ is lower than the one corresponding to $\mathrm{C} 5 \mathrm{CT}$.

\section{Classification}

Regarding classification precision, similar hit rates are yielded by the three alternative approaches (the model yielded by the classification tree algorithm and the two models yielded by the propositional rules algorithm), all proving to be consistent (see Table 8).

In particular, the discriminant model that includes specific costs of incorrect 
Table 6: $\quad$ C5 set of propositional rules ( $\mathrm{C} 5 \mathrm{Ra}$ )

\begin{tabular}{|c|c|c|}
\hline Rules for first time users & & Rules for regular users \\
\hline $\begin{array}{l}\text { IF OPORTO } \\
\text { and AGE }>27 \\
\text { and AGE } \leq 34 \\
\text { and FEMALE } \\
\text { and EDUCATION L. } \leq 4 \\
\text { and INCOME L. } \leq 2 \\
\text { then first time users }(7,0.889) \\
\text { IF AGE } \leq 40 \\
\text { and EDUCATION L. } \leq 4 \\
\text { and INCOME L. } \leq 1 \\
\text { then first time users }(26,0.786) \\
\text { IF OTHER REGIONS } \\
\text { and AGE }>27 \\
\text { and AGE } \leq 40 \\
\text { and [SINGLE or DIVORCED] } \\
\text { and EDUCATION L. }<=4 \\
\text { then first time users }(7,0.778)\end{array}$ & $\begin{array}{l}\text { IF AGE }>24 \\
\text { and AGE } \leq 25 \\
\text { then first time users }(14,0.75) \\
\text { IF AGE }>18 \\
\text { and AGE } \leq 27 \\
\text { and EDUCATION L. } \leq 4 \\
\text { then first time users }(36,0.711) \\
\text { IF AGE }>18 \\
\text { and AGE } \leq 27 \\
\text { and FEMALE } \\
\text { then first time users }(52,0.611) \\
\text { IF AGE } \leq 27 \\
\text { then first time users }(104,0.538)\end{array}$ & $\begin{array}{l}\text { IF } A G E \leq 18 \\
\text { then regular users }(5,0.857) \\
\text { IF AGE } \leq 40 \\
\text { then regular users }(592,0.63) \\
\text { IF AGE }>40 \\
\text { then regular users }(756,0.604)\end{array}$ \\
\hline
\end{tabular}

classification (C5 Rb) shows a better performance for the training sample (specifically, the capacity to classify heavy users correctly is increased). However, for the test sample the results remain similar to the other two models, suggesting that overfitting is the reason for the increase in the classification precision.

The values obtained for global classification precision associated with the demographic profiling variables (see Table 8) should be referred to the original segment distribution which is not uniform (heavy, regular and first time users represent around 20 per cent, 61 per cent and 19 per cent of the pousadas' clients). Thus, one may conclude that:

- the difference between the obtained 62 per cent hit rate and the default precision of classification if a random assignment is considered $(45 \%=$ $20 \%^{2}+61 \%^{2}+19 \%^{2}$ ) represents a 17 per cent improvement;

- the difference between the obtained 62 per cent hit rate and the default precision of classification if a majority default classification rule is adopted represents a 1 per cent improvement (regular users account for 61 per cent of the original sample)

These results represent a slight improvement in default classification precision that deserve some additional comments:

- the choice of profiling variables other than the segmentation base variables, decreases the chance of obtaining very good results from a classification precision point of view. (For example, in the present application C5 CT performed very well when based on segmentation variables $-\mathrm{B}, \mathrm{C}$, CSUP and $\mathrm{CH}$ - yielding hit rates of 99.24 per cent and 97.64 per cent corresponding to the training sample and test sample, respectively.);

- the literature ${ }^{33,34}$ suggests the existence of weak or indirect links between behavioural segments and demographics, which may render profiling based on demographics a difficult task;

- it is reasonable to expect that if alternative discriminant methods 


\section{Cardoso and Moutinho}

Table 7: $\quad$ C5 set of propositional rules (C5 Rb)

\begin{tabular}{|c|c|c|c|}
\hline Rules for first time users & Rules for regular users & Rules for heavy users & \\
\hline $\begin{array}{l}\text { IF AGE }>24 \\
\text { and AGE } \leq 25 \\
\text { then first time users }(14,0.75) \\
\text { IF AGE }>18 \\
\text { and AGE } \leq 27 \\
\text { and EDUCATION L. } \leq 4 \\
\text { then first time users }(36,0.711) \\
\text { IF AGE } \leq 40 \\
\text { and INCOME L. } \leq 1 \\
\text { then first time users }(38,0.675) \\
\text { IF AGE } \leq 53 \\
\text { and EDUCATION L. } \leq 2 \\
\text { then first time users }(22,0.667)\end{array}$ & $\begin{array}{l}\text { IF AGE } \leq 18 \\
\text { then regular users }(5,0.857) \\
\text { IF AGE }>53 \\
\text { and EDUCATION L. } \leq 2 \\
\text { then regular users }(18,0.8) \\
\text { IF OTHER REGIONS } \\
\text { and AGE }>40 \\
\text { and EDUCATION L. } \leq 2 \\
\text { then regular users }(14,0.75) \\
\text { IF AGE }>25 \\
\text { and AGE } \leq 27 \\
\text { and MALE } \\
\text { and EDUCATION L.>4 } \\
\text { then regular users }(24,0.615) \\
\text { IF EDUCATION L.>2 } \\
\text { then regular users }(1324,0.615)\end{array}$ & $\begin{array}{l}\text { IF OTHER REGIONS } \\
\text { and AGE }>67 \\
\text { and EDUCATION L.>2 } \\
\text { then heavy users }(12,0.786) \\
\text { IF AGE }>51 \\
\text { and AGE } \leq 53 \\
\text { and EDUCATION L. } \leq 3 \\
\text { and INCOME L.>3 } \\
\text { then heavy users }(2,0.75) \\
\text { IF AGE }>40 \\
\text { and AGE } \leq 41 \\
\text { and EDUCATION L. }>3 \\
\text { and INCOME L.>3 } \\
\text { then heavy users }(6,0.75) \\
\text { IF OPORTO } \\
\text { and AGE }>53 \\
\text { and EDUCATION L.>4 } \\
\text { and INCOME L.>3 } \\
\text { then heavy users }(5,0.714) \\
\text { IF AGE }>53 \\
\text { and SINGLE } \\
\text { and MALE } \\
\text { and INCOME L. } \leq 3 \\
\text { then heavy users }(4,0.667)\end{array}$ & $\begin{array}{l}\text { IF OPORTO } \\
\text { and AGE }>53 \\
\text { and AGE } \leq 62 \\
\text { and EDUCATION L. } \leq 3 \\
\text { and INCOME L. }<=3 \\
\text { then heavy users }(4,0.667) \\
\text { IF AGE }>53 \\
\text { and DIVORCED } \\
\text { and MALE } \\
\text { and INCOME L. } \leq 3 \\
\text { then heavy users }(1,0.667) \\
\text { IF AGE }>53 \\
\text { and AGE } \leq 70 \\
\text { and WIDOW } \\
\text { and EDUCATION L.>2 } \\
\text { then heavy users }(6,0.625) \\
\text { IF LISBON } \\
\text { and AGE }>53 \\
\text { and MALE } \\
\text { then heavy users }(29,0.548) \\
\text { IF AGE }>64 \\
\text { and MARRIED } \\
\text { and EDUCATION L.>2 } \\
\text { and INCOME L. } \leq 3 \\
\text { then heavy users }(81,0.53)\end{array}$ \\
\hline
\end{tabular}

were used, classification results would be similar. ${ }^{35}$

Finally, it may be concluded that, although the classification precision improvement was only marginal, useful insights were derived from the discriminant logical type models yielded by C5 (propositional rules model, in particular), helping to profile the segment structure.

\section{DISCUSSION}

It has been suggested ${ }^{36}$ that there is some empirical evidence that similar classification precision may be achieved by different discriminant methods, namely linear discriminant analysis, logistic regression, neural networks and classification trees. When choosing an algorithm for profiling a segment structure, additional criteria must, thus, be taken into account. In particular, the need for specific parametric assumptions, the capacity to deal with missing observations, the existence of estimation problems and the interpretability of results, should be considered.

As regards interpretability, a set of propositional rules (particularly when derived by the C5 $\mathrm{R}$ algorithm) speaks for itself. In fact, the use of natural (logical type) language makes the profiles and the structure of classification easy to interpret, thus proving to be very appealing to practitioners.

In addition, some characteristics of the proposed discriminant logical type models (C5 $\mathrm{T}$ and C5 R) may turn out to be an advantage when compared to alternative methods for profiling:

- C5 is a purely nonparametric procedure that requires no assumptions concerning the data, as 
Table 8: Classification precision: C5 classification tree and propositional rules

\begin{tabular}{|c|c|c|c|c|c|c|c|c|c|}
\hline & \multicolumn{9}{|c|}{ Recursive based partitioning models } \\
\hline & \multicolumn{3}{|c|}{ C5 CT } & \multicolumn{3}{|c|}{ C5 Ra } & \multicolumn{3}{|c|}{$\mathrm{C} 5 \mathrm{Rb}$} \\
\hline \multicolumn{2}{|l|}{ Hit rate (training s.) } & \multicolumn{2}{|l|}{$63.3 \%$} & \multicolumn{3}{|c|}{$63.2 \%$} & \multicolumn{3}{|c|}{$64.0 \%$} \\
\hline Actual & $\begin{array}{l}\text { First } \\
\text { time } \\
\text { users }\end{array}$ & $\begin{array}{l}\text { Regular } \\
\text { users }\end{array}$ & $\begin{array}{l}\text { Heavy } \\
\text { users }\end{array}$ & $\begin{array}{l}\text { First } \\
\text { time } \\
\text { users }\end{array}$ & $\begin{array}{l}\text { Regular } \\
\text { users }\end{array}$ & $\begin{array}{l}\text { Heavy } \\
\text { users }\end{array}$ & $\begin{array}{l}\text { First } \\
\text { time } \\
\text { users }\end{array}$ & $\begin{array}{l}\text { Regular } \\
\text { users }\end{array}$ & $\begin{array}{l}\text { Heavy } \\
\text { users }\end{array}$ \\
\hline First time users & 71 & 225 & & 67 & 229 & & 52 & 44 & 0 \\
\hline Regular users & 29 & 847 & & 26 & 850 & & 19 & 854 & 3 \\
\hline Heavy users & 0 & 278 & & 0 & 278 & & 1 & 255 & 22 \\
\hline \multicolumn{2}{|l|}{ Hit rate (test s.) } & \multicolumn{2}{|l|}{$62.0 \%$} & \multicolumn{3}{|c|}{$62.1 \%$} & \multicolumn{3}{|c|}{$61.9 \%$} \\
\hline First time users & 25 & 121 & & 25 & 121 & & 26 & 120 & 0 \\
\hline Regular users & 23 & 448 & & 22 & 449 & & 20 & 441 & 10 \\
\hline Heavy users & 4 & 142 & & 4 & 142 & & 1 & 140 & 5 \\
\hline
\end{tabular}

Note: The numbers in the diagonals correspond to the number of correctly classified cases

opposed to other discriminant methods that may be restricted to obey some assumptions (eg normality in linear discriminant analysis)

- C5 has a specific procedure (summarised in this text) to deal with missing values, as opposed to general statistical discriminant approaches that rely on complete observations for allowing estimation of parameters

- the induction of logical type discriminant models has no estimation problems while some estimation problems may occur when trying to derive statistical models (eg when trying to maximise complex likelihood functions as those that may be associated with mixture models).

Thus, it would be interesting to compare further the proposed approach with alternative discriminant methodologies concerning the above points.

Additionally, the following limitations should also be considered:
- C5 results do not include measures of the descriptors' relative importance for discrimination (typically provided by statistical approaches)

- as the C5 results consistency must be based on cross-validation procedures, the quantity of available data may be a critical issue for the proposed approach.

Finally, further research may also be conducted to compare the performance of the C5 R algorithm with alternative induction of propositional rules algorithms, ${ }^{37}$ for the task of profiling a segment structure.

\section{Acknowledgments}

The first author acknowledges the support of the Department of Business and Management, University of Glasgow in her postdoctoral research.

\section{References}

1 Wind, Y. (1978) 'Issues and advances in segmentation research', Journal of Marketing Research, Vol. XV, pp. 317-337 at p. 318 . 


\section{GLOSSARY}

Classification

Classification tree

Discriminant analysis

Machine learning

Propositional rule

Segment structure

Test set

Training set
Refers to the decision of allocating individuals into classes (eg market segments)

An hierarchical structure that is meant to support the decision of classification. In the root of the tree there are all the observations that base its construction. The leaves represent sub-groups of observations where diversity associated with classes is intended to be minimal

It builds rules (quantitative, logical type or other) that are meant to help differentiating classes. These rules may also be used for classification

It refers to computational algorithms that learn models based on data. Learning with observations is expected to improve with experience (training) in order to yield a better accomplishment of the proposed tasks (eg prediction or classification)

An if condition then class logical type expression where the condition is a conjunctive proposition that allocates specific values to predictors. (Note that a rule may incorrectly classify some observations meeting its condition)

The result from a segmentation process (typically a partition of consumers)

The set of observations that helps to test a model's adequacy The set of observations that base a model's construction
2 Wedel, M. and Kamakura, W. (2000) 'Market segmentation - Conceptual and methodological foundations', Kluwer Academic Publishers, p. 145

3 Mazanec, J. A. (1992) 'Classifying tourists into market segments: A neural network approach', Journal of Travel \& Tourism Marketing, Vol. 1, No. 1, pp. 39-59.

4 Andereck, K. L. and Caldwell, L. L. (1994) 'Variable selection in tourism market segmentation models', Journal of Travel Research, Fall, pp. 40-46.

5 Bargeman, B., Joh, C.-H., Timmermans, H. and Van der Waerden, P. (1999) 'Correlates of tourist vacation behavior: A combination of CHAID and loglinear logit analysis', Tourism Analysis, Vol. 4, pp. 83-93.

6 Mazanec (1992) op. cit.

7 Andereck and Caldwell (1994) op. cit.

8 Bargeman et al. (1999) op. cit.

9 Rao, C. P. and Wang, Z. (1995) 'Evaluating alternative segmentation strategies in standard industrial markets', European Journal of Marketing, Vol. 29, No. 2, pp. 58-75.

10 Bucklin, R. E. and Gupta, S. (1992) 'Brand choice, purchase incidence and segmentation: An integrated modeling approach', Journal of Marketing Research, Vol. 29, No. May, pp. 201-215.

11 Dillon, W. R. Kumar, A. and Borrero, M. S. (1993)
'Capturing individual differences in paired comparisons: An extended BTL model incorporating descriptor variables', Journal of Marketing Research, Vol. 30, February, pp. 42-51.

12 Gupta, S. and Chintagunta, Pk. (1994) 'On using demographic variables to determine segment membership in logic mixture-models', Journal of Marketing Research, Vol. 31, February, pp. 128-136.

13 Mazanec (1992) op cit.

14 Baron, S. and Worral, S. (1996) 'Doing the splits using CHAID to identify segments', OR Insight, Vol. 9, No. 2, pp. 21-25.

15 eg Rao and Wang (1999) op. cit.

16 Press, S. J. and Wilson, S. (1978) 'Choosing between logistic regression and discriminant analysis', Journal of the American Statistical Association, Vol. 73, No. 364, pp. 699-705.

17 Bucklin and Gupta (1992) op. cit.

18 Dillon, W. R. and Kumar, A. (1994) 'Latent structure and other mixture models in marketing: An integrative survey and overview', in Bagozzi, R P. (ed.) 'Advanced methods of marketing research', Blackwell, UK, pp. 295-351.

19 Mazanec (1992) op. cit.

20 Ibid.

21 Bargeman et al. (1999) op. cit.

22 Kass, G. V. (1980) 'An exploratory technique for 
investigating large quantities of categorical data', Applied Statistics, Vol. 29, No. 2, pp. 119-127.

23 Breiman, L., Friedman, J., Olshen, R. and Stone, C. (1984) 'Classification and regression trees', Wadsworth, Inc.

24 Baron and Worrall (1996) op. cit.

25 Bargeman (1999) op. cit.

26 Ratner, B. (1998) 'CHAID for interpreting a logistic regression model', Journal of Targeting, Measurement and Analysis for Marketing, Vol. 6, No. 3, pp. 215-226.

27 Quinlan, J. (1993) 'C4.5: Programs for machine learning’, Morgan Kaufmann Publishers.

28 Cooper, R. A. and Weeks, A. J. (1983) 'Data models and statistical analysis', Philip Allan Publishers Limited.

29 Siegel, S. and Castellan (Jr), N. J. (1988)
'Nonparametric statistics', 2nd edn, McGraw-Hill International Editions.

30 Quinlan (1993) op. cit.

31 Wind (1978) op. cit.

32 Wedel (2000) op. cit.

33 Andereck (1994) op. cit.

34 Ailawadi, K. L., Scott, A. and Gedenk, K. (2001) 'Pursuing the value-conscious consumer: Store brand versus national brand promotions', Journal of Marketing, Vol. 65, pp. 71-89.

35 Thomas, L. T. (2000) 'A survey of credit and behavioural scoring: Forecasting financial risk of lending to consumers', International Journal of Forecasting, Vol. 16, pp. 149-172.

36 Ibid.

37 Clark, P. and Niblett, T. (1988) 'The CN2 induction algorithm', Machine Learning Journal, Vol. 3, No. 4, pp. 261-283. 